\title{
Thermodynamic properties and pressure effect on the superconductivity in CaAlSi and SrAlSi
}

\author{
B. Lorenz, ${ }^{1}$ J. Cmaidalka, ${ }^{1}$ R. L. Meng, ${ }^{1}$ and C. W. Chu ${ }^{1,2,3}$ \\ ${ }^{1}$ Department of Physics and TCSAM, University of Houston, Houston, Texas 77204-5932, USA \\ ${ }^{2}$ Lawrence Berkeley National Laboratory, 1 Cyclotron Road, Berkeley, California 94720, USA \\ ${ }^{3}$ Hong Kong University of Science and Technology, Hong Kong, China \\ (Received 3 February 2003; revised manuscript received 11 April 2003; published 30 July 2003)
}

\begin{abstract}
Superconductivity in the $C 32$ compounds CaAlSi and SrAlSi is investigated by heat capacity measurements as well as high-pressure ac-susceptibility experiments. The heat capacity in the superconducting state is well explained by the BCS theory for weak (SrAlSi) and enhanced coupling strength (CaAlSi). The magnitudes of the superconducting gaps are estimated as $2.7 \mathrm{meV}(\mathrm{CaAlSi})$ and $1.4 \mathrm{meV}$ (SrAlSi). The pressure effect on the superconducting transition temperature, $d \ln T_{c} / d p$, is positive in CaAlSi $\left(+0.026 \mathrm{GPa}^{-1}\right)$ but negative in SrAlSi $\left(-0.024 \mathrm{GPa}^{-1}\right)$. The opposite sign of the pressure coefficients of $T_{c}$ indicates distinct differences in the electronic structure and the density of states at the Fermi energy of the two compounds in agreement with recent band structure calculations.
\end{abstract}

DOI: $10.1103 /$ PhysRevB.68.014512

PACS number(s): 74.25.Bt, 74.62.Fj, 74.70.Ad

\section{INTRODUCTION}

Since the discovery of superconductivity at $39 \mathrm{~K}$ in $\mathrm{MgB}_{2}$, the attention has turned to other intermetallic compounds with similar structure or lattice symmetry. Besides $\mathrm{MgB}_{2}$ only a few binary compounds between the IIA and IIIB elements crystallize in the hexagonal $C 32$ structure, i.e., $\mathrm{CaGa}_{2}, \mathrm{SrGa}_{2}$, and $\mathrm{BaGa}_{2}$. These gallium-based binary compounds are not superconducting. ${ }^{1}$ However, a new class of pseudoternary compounds with $C 32$ structure, $\mathrm{AX}_{2-x} \mathrm{Si}_{x}$ with $A=\mathrm{Ca}, \mathrm{Sr}, \mathrm{Ba}$ and $X=\mathrm{Ga}, \mathrm{Al}$, was recently synthesized and characterized. ${ }^{1-6}$ The pseudoternary compounds are derived from the binary ones by partially substituting $X$ (e.g., $\mathrm{Ga}$ ) by silicon, which results in a change of the lattice parameters ${ }^{1,4}$ and, presumably, of the electronic structure. The compounds are isostructural to $\mathrm{MgB}_{2}$ where the $A$ ions occupy the $\mathrm{Mg}$ sites and the $\mathrm{X} / \mathrm{Si}$ ions are randomly distributed among the boron sites in the honeycomb planes of the C32 structure. The search for superconductivity was successful for most of the materials mentioned above; however, stoichiometric BaAlSi is not superconducting above $2 \mathrm{~K}$ (the measuring limit of previous investigations). Magnetization measurements show the characteristic features of type II superconductors. ${ }^{2}$ The $C 32$ structure is stable within a wide range of $\mathrm{Al}: \mathrm{Si}$ and $\mathrm{Ga}: \mathrm{Si}$ ratios and superconductivity was observed in a range from 0.6 to $2.3(\mathrm{Al}: \mathrm{Si})$ and 0.3 to 1.9 (Ga:Si), respectively. ${ }^{1,4}$ The maximum $T_{c}$ 's were found near the stoichiometric 1:1:1 composition $(x=1)$ in $\mathrm{CaAl}_{2-x} \mathrm{Si}_{x}, \mathrm{SrGa}_{2-x} \mathrm{Si}_{x}$, and $\mathrm{BaGa}_{2-x} \mathrm{Si}_{x}$ but at $x=0.85$ in $\mathrm{CaGa}_{2-x} \mathrm{Si}_{x}$.

Because of the structural similarity to the famous sister compound, $\mathrm{MgB}_{2}$, the question has been raised whether the properties and mechanisms leading to superconductivity in the pseudoternary compounds are of the same origin as those in magnesium diboride and why their $T_{c}$ is so much lower (the highest $T_{c} \approx 7.9 \mathrm{~K}$ was found in CaAlSi). We have shown, based on thermoelectric power measurements, that the majority of charge carriers in the pseudoternary compounds are electron-like in contrast to the $\sigma$-hole carriers in
$\mathrm{MgB}_{2} \cdot{ }^{1,4}$ This conclusion is supported by recent band structure calculations showing that the electron-like $d$ states of the alkaline-earth metals contribute up to $60 \%$ to the total density of states at the Fermi energy. ${ }^{7}$

It is interesting to note that there is no obvious correlation of $T_{c}$ with the mass of the alkaline-earth-metal ion in the compound $A-\mathrm{GaSi}$. However, in $A-\mathrm{AlSi} \mathrm{T}_{c}$ decreases systematically with increasing mass of the $A$ ion from $7.9 \mathrm{~K}$ $(A=\mathrm{Ca})$ to $5 \mathrm{~K}(A=\mathrm{Sr})$ and BaAlSi is not superconducting above $2 \mathrm{~K}^{4,6}$ Imai et al. ${ }^{6}$ attributed the trend of $T_{c}$ in the $A$-AlSi system to a decrease of the density of states, $N\left(E_{F}\right)$, due to the increasing mass of the $A$ ion assuming thereby that the Debye temperature (the prefactor in the BCS formula for $T_{c}$ ) does not change if $\mathrm{Ca}$ is replaced by $\mathrm{Sr}$ and $\mathrm{Ba}$. This assumption, however, is not justified because, unlike in $\mathrm{MgB}_{2}$, the electron-phonon interaction leading to the formation of Cooper pairs and superconductivity is expected to involve the vibrations of the alkaline-earth-metal ions and their mass should affect the superconducting $T_{c}$. The latter argument is supported by the results of recent band structure calculations for $A$-AlSi indicating that $N\left(E_{F}\right)$ actually increases if $\mathrm{Ca}$ is replaced by $\mathrm{Sr}$ or $\mathrm{Ba}^{7}$ Furthermore, the alkaline-earth-metal $d$ states contribute to the Fermi surface to a large extent and there is no reason to assume that they do not participate in the electron-phonon coupling. Additional investigations are needed to reveal the physical nature of the superconducting state.

We, therefore, have conducted measurements of the specific heat as well as the effect of hydrostatic pressure on the superconducting transitions of CaAlSi and SrAlSi. The heat capacity data are useful to (i) measure the thermodynamic properties and confirm the bulk nature of superconductivity, (ii) compare the specific heat in the normal and superconducting states with the standard BCS theory, ${ }^{8,9}$ and (iii) extract microscopic parameters, e.g., the superconducting gap. The application of pressure is of interest because an extraordinary large superconducting $T_{c}$ of $14 \mathrm{~K}$ was reported for a high pressure phase of the binary silicide $\mathrm{CaSi}_{2} .{ }^{10}$

The heat capacity data clearly show the existence of bulk 
superconductivity in CaAlSi and $\mathrm{SrAlSi}$, consistent with previous magnetic measurements. ${ }^{1,2}$ The temperature dependence of the specific heat of SrAlSi fits well the BCS theory in the weak-coupling limit. Deviations from the BCS model in the case of CaAlSi indicate a slightly stronger coupling. With the application of hydrostatic pressure $T_{c}$ increases in CaAlSi but it decreases in SrAlSi. The opposite signs of the pressure coefficients of $T_{c}$ are explained in terms of the distinct difference between the electronic structures of both compounds.

\section{EXPERIMENT}

The pseudoternary intermetallic compounds, CaAlSi and SrAlSi, have been synthesized by argon arc melting with appropriate amounts of $\mathrm{Ca}(99 \%), \mathrm{Sr}(99 \%), \mathrm{Al}(99.99 \%)$, and $\mathrm{Si}(99.99 \%)$ as described earlier. ${ }^{1}$ The x-ray powder diffraction spectra show the $C 32$ hexagonal structure with lattice constants $(a, c)=(4.189,4.400 \AA)$ for $\mathrm{CaAlSi}$ and $(a, c)=(4.220,4.754 \AA)$ for SrAlSi. Magnetization and transport measurements indicate the onset of superconductivity at $7.9 \mathrm{~K}$ and $5.0 \mathrm{~K}$ in CaAlSi and SrAlSi, respectively. ${ }^{1,4}$

The heat capacity was measured for both samples (typical mass of $25 \mathrm{mg}$ ) between room temperature and $2.2 \mathrm{~K} \mathrm{em}-$ ploying the Physical Property Measurement System (Quantum Design). The relative error of the measurement (i.e., the statistical error of the fitting procedure) was of the order of 0.1 to $0.2 \%$. The absolute accuracy of the measurement was tested in particular at low temperatures by comparing the measured heat capacity of high purity (99.9999\%) lead with the available data. ${ }^{11}$ The agreement of the lead measurement with the literature data was within the statistical data fluctuation.

The pressure effect on the superconducting transitions of $\mathrm{CaAlSi}$ and SrAlSi was investigated by ac-susceptibility measurements at pressures up to $2 \mathrm{GPa}$. A dual coil system was mounted directly to the sample. The ac susceptibility was measured using the mutual inductance bridge (LR700, Linear Research). Pressure was generated in a berylliumcopper piston-cylinder clamp. ${ }^{12}$ The sample was mounted in a Teflon container filled with a 1:1 mixture of Fluorinert FC70 and FC77 as a hydrostatic pressure transmitting medium. The pressure was measured in situ at $7 \mathrm{~K}$ by monitoring the shift of the superconducting $T_{c}$ of a high-purity (99.9999\%) lead manometer. The temperature above $45 \mathrm{~K}$ was measured by a thermocouple inside the Teflon container and, at low temperatures, by a germanium resistor built into the pressure cell close to the sample position. Data have been taken upon loading and unloading cycles.

\section{RESULTS AND DISCUSSION}

\section{A. Heat capacity measurements}

Figures 1(a) and (b) show the low temperature heat capacity data, $C_{p} / T$ vs $T$, for CaAlSi and SrAlSi, respectively. At zero magnetic field (open circles) $C_{p}$ shows a sharp peak at the superconducting transition as expected for bulk superconductors with a narrow transition width of $0.4 \mathrm{~K}$ (CaAlSi) and $0.2 \mathrm{~K}$ (SrAlSi). To compare with the normal state heat

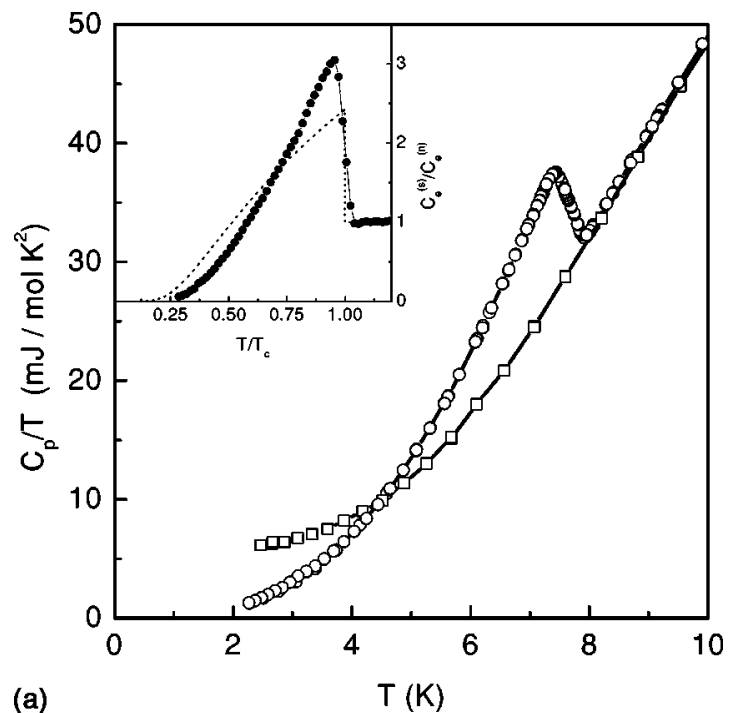

(a)

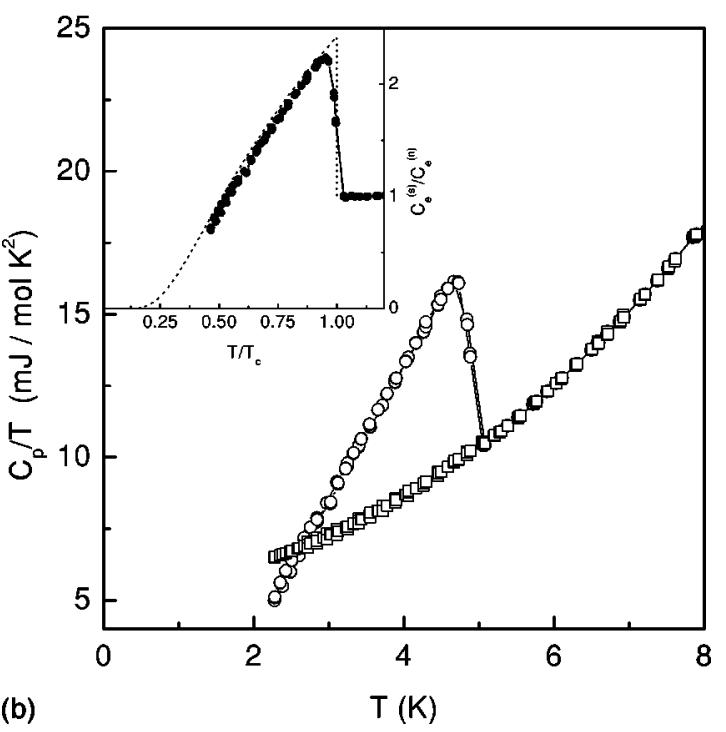

FIG. 1. Low temperature heat capacity of (a) CaAlSi and (b) SrAlSi. The open circles show $C_{p}$ measured in zero magnetic field. The normal state $C_{p}$ (open squares) was measured by suppressing superconductivity in a magnetic field of $7 \mathrm{~T}$. The insets show the plot of the electronic specific heat in the superconducting state, $C_{e}^{(s)} / C_{e}^{(n)}$ vs $T / T_{c}$, with the BCS function (dotted line).

capacity, the superconductivity was suppressed by a $7 \mathrm{~T}$ magnetic field. The corresponding data are shown by open squares in Fig. 1. The high field data can be used to estimate the superconducting component of $C_{p}$ by subtracting the lattice contribution from the zero field $C_{p}$ below $T_{c}$. In general $C_{p}$ is given by the sum of the electronic $\left(C_{e}\right)$ and the lattice $\left(C_{l}\right)$ contributions

$$
C_{p}(T)=C_{e}(T)+C_{l}(T),
$$

with $C_{e}(T)=C_{e}^{(n)}(T)=\gamma T$ in the normal state and $C_{e}(T)$ $=C_{e}^{(s)}(T)$ in the superconducting state. The values of $C_{e}^{(s)}(T)$ in the BCS theory for weak coupling were numerically calculated and tabulated by Mühlschlegel. ${ }^{8}$ The characteristic coefficient $\gamma$ of $C_{e}^{(n)}$ is proportional to the density of 
states (DOS) at the Fermi energy, $N\left(E_{F}\right)$. The lattice heat capacity at low temperatures can be expanded in a power series of $T$ (Debye model) starting with the third order term, $T^{3}$. Normal state electronic and lattice contributions are commonly separated in the $C_{p} / T$ vs $T^{2}$ plot where the coefficient $\gamma$ is obtained as the linear extrapolation of $C_{p} / T$ to zero temperature and the slope at low temperatures is used to calculate the Debye temperature, $\Theta_{D}$. Using the high field data of $C_{p}$ we estimate $\gamma=5.04 \mathrm{~mJ} / \mathrm{mol} \mathrm{K}, \Theta_{D}=226 \mathrm{~K}$, and $\gamma=5.48 \mathrm{~mJ} / \mathrm{mol} \mathrm{K}, \Theta_{D}=218 \mathrm{~K}$ for CaAlSi and SrAlSi, respectively. It is interesting to note that $\gamma$ of SrAlSi is indeed larger than the value for CaAlSi indicating that $N\left(E_{F}\right)$ increases with the substitution of $\mathrm{Ca}$ by $\mathrm{Sr}$ in qualitative agreement with the band structure calculations. ${ }^{7}$ However, the increase is moderate and far smaller than the factor of two predicted by the theory.

The electronic heat capacity in the superconducting state, $C_{e}^{(s)}(T)$, is compared with the BCS data (dotted line) in the insets of Fig. 1. The thermodynamic $T_{c}$ was estimated by an entropy conserving construction as $T_{c}=7.7 \mathrm{~K}(\mathrm{CaAlSi})$ and $T_{c}=4.9 \mathrm{~K}(\mathrm{SrAlSi})$. These values are slightly lower than the values determined from resistivity measurements or from the onset of the diamagnetic drop of the magnetic susceptibility. However, this is to be expected since the thermodynamic $T_{c}$ represents an averaged bulk value whereas resistivity as well as the onset of diamagnetism are both sensitive to the first percolating path of superconducting volume in the sample. The normalized electronic heat capacity, $C_{e}^{(s)}(T) / C_{e}^{(n)}(T)$, is displayed as a function of $T / T_{c}$. There is an excellent agreement with the BCS data in the case of SrAlSi [Fig. 1(b)], i.e., the BCS model in the weak-coupling limit describes the superconducting state very well in the temperature range accessible in our experiments $(T>2.2 \mathrm{~K})$. There are deviations of $C_{e}^{(s)}(T)$ from the BCS data in the case of CaAlSi. The heat capacity falls below the BCS values at low $T$ but is enhanced close to $T_{c}$ resulting in a larger relative jump of $C_{p}, \delta C_{p}\left(T_{c}\right) / \gamma T_{c} \approx 2$ (BCS value $=1.43$ ). These deviations may be explained by a stronger electron phonon coupling in CaAlSi as compared to SrAlSi. The thermodynamic consistency of the heat capacity data was checked by integrating the entropy difference, $\left[C_{e}^{(s)}(T)-C_{e}^{(n)}(T)\right] / T$, from $T=0$ to $T=T_{c}$. For CaAlSi this integral is close to zero as expected. For SrAlSi the integration cannot be carried out with sufficient accuracy because of the experimental restrictions (the lowest accessible temperature, $2.2 \mathrm{~K}$, is just little less than $50 \%$ of $T_{c}$ ). The superconducting gap at zero temperature, $2 \Delta$, may be estimated from the heat capacity at low temperatures by plotting $\ln C_{e}^{(s)}(T)$ vs $1 / T$ (Arrhenius plot). The low temperature slopes estimated from this plot yield values of $2 \Delta=1.4 \mathrm{meV}$ and $2 \Delta=2.7 \mathrm{meV}$ for SrAlSi and CaAlSi, respectively. However, because of the limited temperature range of our data $(T>2.2 \mathrm{~K})$ these values are certainly underestimated and should be considered as a lower limit of the gap parameter. A more accurate estimate requires heat capacity measurements at far lower temperatures. The BCS value of the gap is related to $T_{c}$ by $2 \Delta_{B C S}=3.53 k_{B} T_{c}$. For SrAlSi with $T_{c}=4.9 \mathrm{~K}$ we get $2 \Delta_{B C S}=1.49 \mathrm{meV}$, which is only slightly larger than our estimate. Taking into account
TABLE I. The characteristic normal and superconducting state parameters of CaAlSi and SrAlSi as compared to the weak coupling BCS theory.

\begin{tabular}{lccc}
\hline \hline & CaAlSi & SrAlSi & BCS theory \\
\hline$\gamma\left(\mathrm{mJ} / \mathrm{mol} \mathrm{K}^{2}\right)$ & 5.04 & 5.42 & \\
$\theta_{D}(\mathrm{~K})$ & 226 & 218 & \\
$T_{c}(\mathrm{~K})$ & 7.7 & 4.9 & \\
$2 \Delta(\mathrm{meV})$ & 2.7 & 1.49 & \\
$\delta C_{p}\left(T_{c}\right) / \gamma T_{c}$ & 2.0 & 1.4 & 1.43 \\
$2 \Delta / k_{B} T_{c}$ & 4.07 & $\sim 3.5$ & 3.53 \\
\hline \hline
\end{tabular}

the almost perfect agreement of $C_{e}^{(s)}(T)$ with the BCS function over the full experimental temperature range, the superconducting gap value for SrAlSi can be assumed to be close to the BCS value of $1.49 \mathrm{meV}$. However, the calculated BCS gap parameter for CaAlSi, $2 \Delta_{B C S}=2.34 \mathrm{meV}$, is clearly smaller than the value of $2.7 \mathrm{meV}$ estimated as a lower limit of $2 \Delta$ from the Arrhenius plot of $C_{e}^{(s)}(T)$. This enhancement of the gap with respect to the BCS parameter is a further indication of an increased electron-phonon coupling. A summary of all relevant parameters and the comparison to the BCS weak coupling values is given in Table I.

The temperature dependence of the heat capacity in the superconducting state provides strong evidence that the superconductivity found in SrAlSi and CaAlSi is well explained by the standard BCS theory for weak and enhanced coupling, respectively. Furthermore, the data confirm the bulk nature of the superconductivity in these pseudoternary C32 compounds.

It appears interesting to compare the present results with the superconductivity found at relatively high temperatures in binary compounds with similar structure. Superconductivity at $14 \mathrm{~K}$ was recently detected in $\mathrm{CaSi}_{2}$ at pressures above $14 \mathrm{GPa}^{10}$ The (tetragonal) structure of this high-pressure phase is very similar to the $\mathrm{AlB}_{2}$ structure but the honeycomb planes of the $\mathrm{Si}$ ions show still a nonvanishing buckling even at the highest pressures. ${ }^{13}$ No thermodynamic data such as heat capacity are known for this superconducting high-pressure phase. In contrast, extensive research has been conducted to investigate the thermodynamic properties in the superconducting state of $\mathrm{MgB}_{2}$. The heat capacity of $\mathrm{MgB}_{2}$ shows an abnormal temperature dependence below $T_{c} .{ }^{14}$ Whereas the value of $C_{e}^{(s)}(T)$ at and right below $T_{c}$ is smaller than the corresponding BCS function, it raises above the BCS data below about $20 \mathrm{~K}$ followed by an exponential drop at lower temperature. From this unusual behavior the existence of two superconducting gaps in $\mathrm{MgB}_{2}$ was suggested and later confirmed by many alternative experiments. The present data for CaAlSi and SrAlSi show no evidence for a similar scenario within the experimental temperature range of $T>2.2 \mathrm{~K}$, i.e., $T>0.44 T_{c}$ for $\mathrm{SrAlSi}$ and $T$ $>0.27 T_{c}$ for CaAlSi. The existence of an anomaly at far lower temperature, similar to $\mathrm{MgB}_{2}$, cannot be completely excluded from the current investigation. However, the almost perfect agreement of $C_{e}^{(s)}(T)$ with the BCS data in the case 

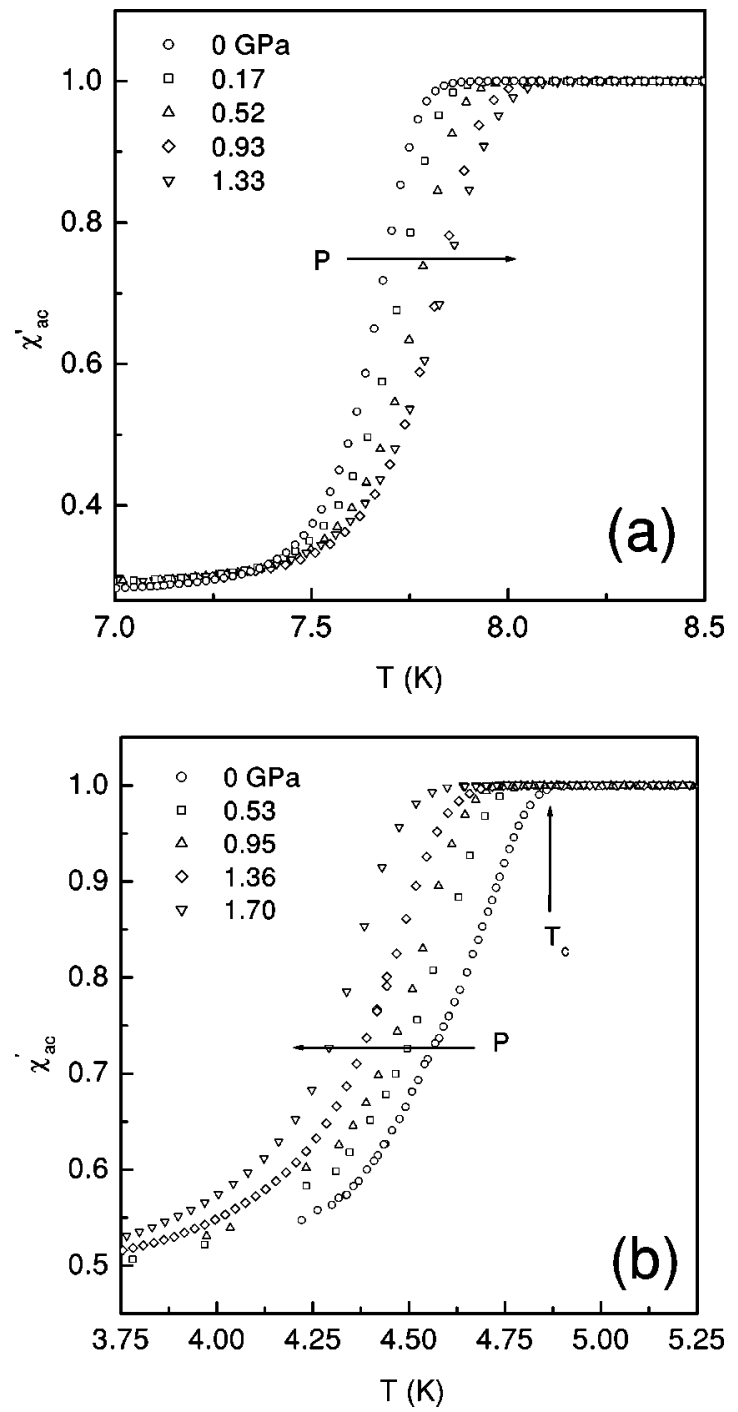

FIG. 2. ac susceptibility of (a) CaAlSi and (b) SrAlSi measured at different pressures. The data are normalized to the susceptibility value right above $T_{c}$. The superconducting transition temperature was estimated from the onset of the diamagnetic signal.

of SrAlSi as well as the enhancement of $C_{e}^{(s)}(T)$ close to $T_{c}$ of CaAlSi (instead of the depletion observed in $\mathrm{MgB}_{2}$ ) suggest a less complex structure of the superconducting gap as compared to magnesium diboride.

\section{B. Pressure effect on the superconducting transitions in CaAlSi and SrAISi}

The effect of hydrostatic pressure on the superconducting $T_{c}$ is of special interest since it can help to reveal the intrinsic mechanisms of superconductivity. In $\mathrm{MgB}_{2}$, for example, it could be shown that the negative pressure coefficient of $T_{c}$ was most compatible with the strong-coupling model (McMillen) of phonon-mediated superconductivity so that alternative explanations appeared to be less favorable. ${ }^{15}$

The pressure effect on $T_{c}$ of CaAlSi and SrAlSi was investigated by measuring the real part of the ac susceptibility, $\chi_{a c}^{\prime}$. The data in Fig. 2 show the shift of the diamagnetic
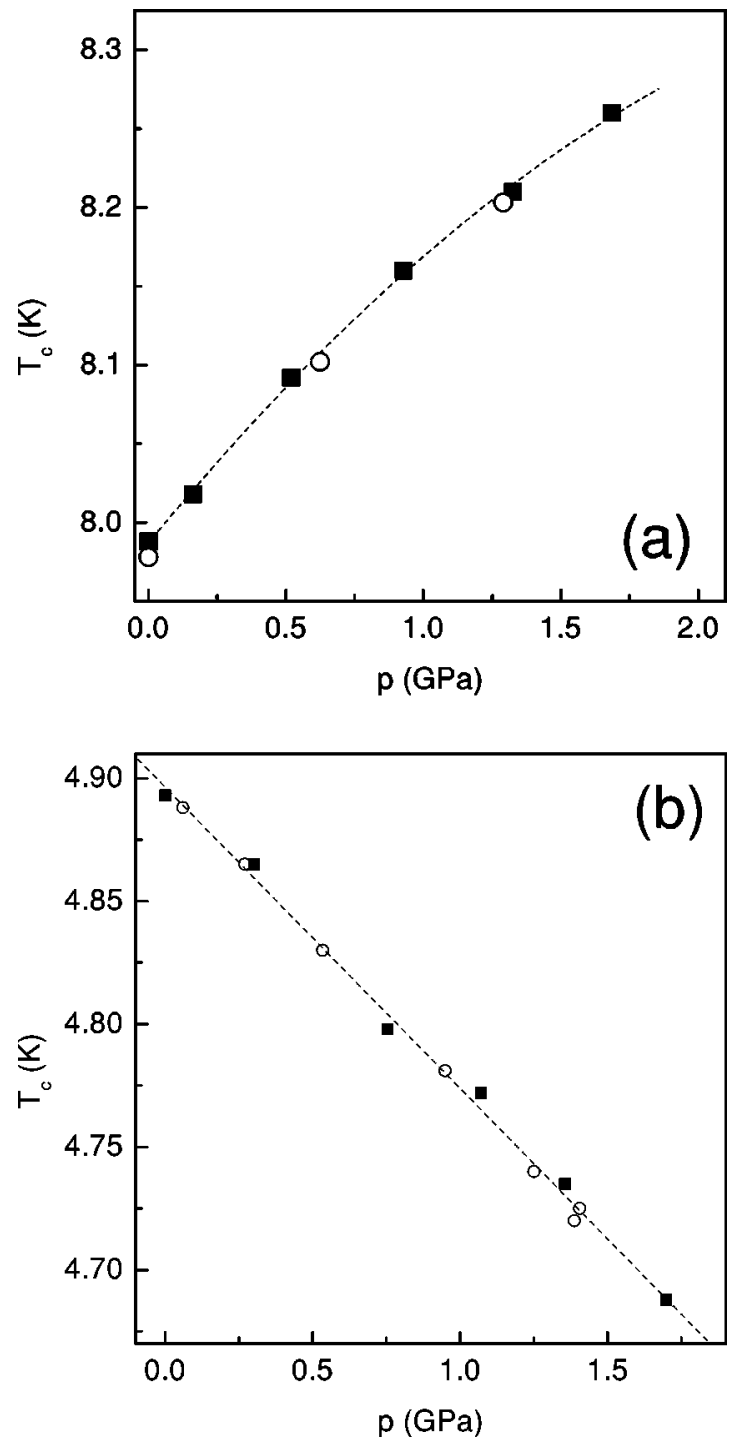

FIG. 3. Pressure dependence of $T_{c}$ of (a) CaAlSi and (b) SrAlSi. The filled squares and open circles are data taken at increasing and decreasing pressure, respectively.

signal of $\chi_{a c}^{\prime}$ with increasing pressure. Surprisingly, the pressure effect on CaAlSi and SrAlSi is opposite in sign. The superconducting transition temperature (measured at the onset of the diamagnetic signal) of CaAlSi increases with pressure, whereas $T_{c}$ of SrAlSi clearly decreases. The $T_{c}$ 's as a function of pressure for both compounds are shown in Fig. 3. The increase of $T_{c}$ in CaAlSi [Fig. 3(a)] is slightly nonlinear with an initial slope of $0.21 \mathrm{~K} / \mathrm{GPa}$. In contrast, Fig. 3(b) shows an almost perfect linear decrease of $T_{c}$ for SrAlSi with a coefficient of $-0.12 \mathrm{~K} / \mathrm{GPa}$. Although the magnitude of the relative pressure coefficient, $d \ln \left(T_{c}\right) / d p$, is almost the same for both compounds, the opposite sign deserves further consideration.

For a qualitative discussion we use the BCS equation for $T_{c}$ (Ref. 9)

$$
k_{B} T_{c}=1.13 \hbar \omega_{D} \exp (-1 / \lambda)
$$


where $\hbar \omega_{D}$ is a measure of the characteristic energy scale for the phonons and $\lambda=V_{0} N\left(E_{F}\right) . V_{0}$ is the matrix element of the effective interaction. Assuming that the pressure dependence of $V_{0}$ is negligible, the pressure coefficient of $T_{c}$ is derived as

$$
\frac{d \ln T_{c}}{d p}=\frac{d \ln \omega_{D}}{d p}+\frac{1}{V_{0} N\left(E_{F}\right)} \frac{d \ln V_{0} N\left(E_{F}\right)}{d p} .
$$

There are obviously two contributions to the pressure coefficient of $T_{c}$ : one from the sole phonon system, $d \ln \omega_{D} / d p$, and the second one involving the electronic system, $d \ln N\left(E_{F}\right) / d p$. In the weak-coupling limit of the BCS model both terms contribute to the total pressure coefficient. The effect of pressure on the phonon system usually results in an increase of the average phonon energy, i.e., in a "hardening" of phonon modes. Only in rare cases, close to a structural transition, phonon "softening" may occur but usually only for some particular modes. Therefore, the first term in Eq. (3) yields a positive contribution to the total pressure coefficient. The pressure effect on the density of states, however, is more complex. The compression of the lattice by pressure in general causes an increase of the bandwidth, which in turn will result in an average decrease of $N(E)$ since the total number of states in a band is fixed. In addition, $V_{0}$ tends to decrease with pressure since it is inversely proportional to the average square of the phonon frequency. This negative contribution to $d \ln T_{c} / d p$ will compete with the phonon hardening effect. However, the application of pressure may also cause slight changes in the band structure and/or a shift of the Fermi energy, $E_{F}$, and a change of $N\left(E_{F}\right)$. Therefore, within the BCS theory the pressure coefficient of $T_{c}$ can be of either sign, depending on which of the contributions to Eq. (3), positive or negative, dominate.

In both compounds, CaAlSi and SrAlSi, the phonon contribution to the pressure coefficient is expected to be positive. The magnitude of $d \ln \omega_{D} / d p$ is not known so far but it can be estimated from Raman or infrared spectroscopy at high pressures. The electronic term in Eq. (3) needs a careful consideration. Recent band structure calculations have shown striking differences in $N(E)$ in CaAlSi and SrAlSi. ${ }^{7}$ In CaAlSi the Fermi energy lies in a region where $N(E)$ is flat and relatively insensitive to small variations of $E_{F}$. This results in a small electronic contribution to the total pressure coefficient of $T_{c}$. In CaAlSi the positive phonon term obviously outweighs the electronic term, which results in the observed positive $d T_{c} / d p$, as shown in Fig. 3(a). The situation is different, however, in SrAlSi. According to the calculations ${ }^{7}$ the density of states forms a very narrow peak close to the Fermi energy. This makes $N\left(E_{F}\right)$ extremely susceptible to external perturbations. Any small change induced by pressure (band broadening, shift of $E_{F}$ ) may result in a stronger decrease of $N\left(E_{F}\right)$. The negative electronic contribution to the pressure coefficient of $T_{c}$, therefore, can dominate over the lattice term and the total $d T_{c} / d p$ becomes negative as observed in our experiments [Fig. 3(b)].

Although the above discussion is qualitative in nature it indicates that the opposite pressure coefficients of $T_{c}$ in $\mathrm{CaAlSi}$ and SrAlSi uniquely reflect the peculiarities of their electronic structure. They provide an indirect proof of the distinct differences in the density of states at $E_{F}$ in both compounds, CaAlSi and $\mathrm{SrAlSi}$, as suggested by band structure calculations. Furthermore, the positive pressure coefficient observed in CaAlSi cannot be explained by a sole change of the density of states induced by pressure but the contribution due to phonon hardening has to be taken into account. For a more quantitative comparison additional experimental as well as theoretical work is needed. The measurement of phonon frequencies as a function of pressure should provide a better estimate of the phonon effect on $d \ln T_{c} / d p$. Improved band structure calculations involving the effects of lattice compression and including the phonon system (see, for example, Ref. 16) may help to get a more adequate understanding of the superconductivity in the pseudobinary $C 32$ intermetallic compounds.

\section{SUMMARY AND CONCLUSIONS}

We have investigated the superconductivity in the $C 32$ intermetallic compounds CaAlSi and SrAlSi by heat capacity and high-pressure ac susceptibility measurements. Several parameters characterizing the normal and superconducting states are extracted from the heat capacity. The coefficients of the normal state electronic specific heat are estimated as $\gamma=5.04 \mathrm{~mJ} / \mathrm{mol} \mathrm{K}^{2}$ and $\gamma=5.42 \mathrm{~mJ} / \mathrm{mol} \mathrm{K}^{2}$ for CaAlSi and $\mathrm{SrAlSi}$, respectively. The values of the superconducting gap are calculated from the electronic heat capacity in the superconducting state as $2.7 \mathrm{meV}(\mathrm{CaAlSi})$ and $1.4 \mathrm{meV}(\mathrm{SrAlSi})$. The parameters are consistent with the predictions of the BCS theory in the weak-coupling limit for SrAlSi and for enhanced coupling in the case of CaAlSi. The bulk nature of superconductivity in CaAlSi and SrAlSi is confirmed.

The effect of hydrostatic pressure on $T_{c}$ is positive in the case of CaAlSi $\left(d \ln T_{c} / d p=0.026 \mathrm{GPa}^{-1}\right)$ but negative for SrAlSi $\left(d \ln T_{c} / d p=-0.024 \mathrm{GPa}^{-1}\right)$. This opposite tendency of the pressure shift of $T_{c}$ reflects the distinct differences in the density of states near the Fermi energy in both compounds. The high-pressure data also underline the importance of the phonon frequency (or ionic mass) for understanding the superconductivity in the system $A-\mathrm{AlSi}$ and, in particular, the positive pressure effect observed in CaAlSi. With the increasing mass of the alkaline-earth ion $T_{c}$ is systematically suppressed, from $7.7 \mathrm{~K}(\mathrm{Ca})$ to $4.9 \mathrm{~K}(\mathrm{Sr})$ and to nonsuperconducting above $2 \mathrm{~K}(\mathrm{Ba})$. This tendency cannot be explained as a sole density-of-states effect since our data show that $N\left(E_{F}\right)$ increases when $\mathrm{Ca}$ is replaced by $\mathrm{Sr}$, in qualitative agreement with the band structure calculations.

\section{ACKNOWLEDGMENTS}

This work was supported in part by NSF Grant No. DMR9804325, the T.L.L. Temple Foundation, the John J. and Rebecca Moores Endowment, and the State of Texas through the TCSAM at the University of Houston and at Lawrence Berkeley Laboratory by the Director, Office of Energy Research, Office of Basic Energy Sciences, Division of Materials Sciences of the U.S. Department of Energy under Contract No. DE-AC03-76SF00098. 
${ }^{1}$ R. L. Meng, B. Lorenz, Y. S. Wang, J. Cmaidalka, Y. Y. Sun, Y. Y. Xue, J. K. Meen, and C. W. Chu, Physica C 382, 113 (2002); R. L. Meng, B. Lorenz, J. Cmaidalka, Y. S. Wang, Y. Y. Sun, J. Lenzi, J. K. Meen, Y. Y. Xue, and C. W. Chu, Physica C (to be published).

${ }^{2}$ M. Imai, E. Abe, J. Ye, K. Nishida, T. Kimura, K. Honma, H. Abe, and H. Kitazawa, Phys. Rev. Lett. 87, 077003 (2001).

${ }^{3}$ M. Imai, K. Noshida, T. Kimura, and H. Abe, Appl. Phys. Lett. 80, 1019 (2002).

${ }^{4}$ B. Lorenz, J. Lenzi, J. Cmaidalka, R. L. Meng, Y. Y. Sun, Y. Y. Xue, and C. W. Chu, Physica C 383, 191 (2002).

${ }^{5}$ M. Imai, K. Nishida, T. Kimura, and H. Abe, Physica C 377, 96 (2002).

${ }^{6}$ M. Imai, K. Nishida, T. Kimura, H. Kitazawa, H. Abe, H. Kito, and K. Yoshii, Physica C 382, 361 (2002).

${ }^{7}$ I. R. Shein, N. I. Medvedeva, and A. L. Ivanovskii, cond-mat/0212047 (unpublished).

${ }^{8}$ B. Mühlschlegel, Z. Phys. 155, 313 (1959).
${ }^{9}$ C. P. Poole, Jr., H. A. Farach, and R. J. Creswick, Superconductivity (Academic Press, New York, 1996).

${ }^{10}$ S. Sanfilippo, H. Elsinger, M. Nunez-Regueiro, O. Laborde, S. LeFloch, M. Affronte, G. L. Olcese, and A. Palenzona, Phys. Rev. B 61, R3800 (2000).

${ }^{11}$ M. Horowitz, A. A. Silvidi, S. F. Malaker, and J. G. Daunt, Phys. Rev. 88, 1182 (1952); B. J. C. van der Hoeven, Jr. and P. H. Keesom, Phys. Rev. 137, A103 (1965).

${ }^{12}$ C. W. Chu and L. R. Testardi, Phys. Rev. Lett. 32, 766 (1974).

${ }^{13}$ P. Bordet, M. Affronte, S. Sanfilippo, M. Nunez-Regueiro, O. Laborde, G. L. Olcese, A. Palenzona, S. LeFloch, D. Levy, and M. Hanfland, Phys. Rev. B 62, 11392 (2000).

${ }^{14}$ F. Bouquet, R. A. Fisher, N. E. Phillips, D. G. Hinks, and J. D. Jorgensen, Phys. Rev. Lett. 87, 047001 (2001).

${ }^{15}$ B. Lorenz, R. L. Meng, and C. W. Chu, Phys. Rev. B 64, 012507 (2001).

${ }^{16}$ I. Loa and K. Syassen. Solid State Commun. 118, 575 (2001). 